\title{
Effect of aging in artificial saliva on the shear bond strength of resin composite
}

SADJ November 2018, Vol. 73 No. 10 p617 - p622

KW Irari', DS Moodley², N Patel ${ }^{3}$

\section{ABSTRACT}

\section{Introduction}

Artificial saliva as an in vitro aging medium may be an effective method of simulating the clinical degradation of resin composite restorations. The composition and desirable properties of artificial saliva are not yet standardised. Incorporation of oral bacteria may more closely approximate the intra-oral effect of saliva on composite restorations. Surface deterioration on an old broken restoration repaired with fresh composite may result in a weak adhesive interface, measureable, in vitro, by a shear bond strength test.

\section{Aim of the study}

To evaluate whether artificially aged composite affects the shear bond strength of the adhesive interface with fresh composite.

\section{Materials and methods}

A silicone mould was used to fabricate 240 composite cylinders. 120 were aged in a solution of artificial saliva for 28 days. The remainder served as controls. The specimens were divided into seven groups of twenty, each group being subjected to a different repair protocol. Shear bond strength testing was then carried out on the repaired composite blocks.

\section{Results}

Aging in artificial saliva resulted in a reduction of $15.04 \%$ to $21.1 \%$ in shear bond strengths compared with un-aged composite.

\section{Conclusion}

Composite samples aged in artificial saliva had a significantly weaker repair interface compared with nonaged samples.

\section{Author affiliations:}

1. Ken W. Irari, B.D.S. (UoN), MSc. Restorative Dentistry (U.W.C.), Moi University School of Dentistry, Eldoret, Kenya. Principal researcher.

2. Desi S. Moodley, PhD, MSc Dent; PDD Aesth; BDS,

Senior Lecturer, Dept. of Restorative Dentistry University of the Western Cape. Research supervisor, co-author.

3. Naren Patel, MChD; BDS; PDD Implantology,

Specialist Prosthodontist, Dept. of Restorative Dentistry University of the Western Cape. Co-author.

Corresponding author: Ken W. Irari

Moi University School of Dentistry, Eldoret, Kenya.

Cell: +254 (0)739209748

Email: kenirari@mu.ac.ke/kenirari@gmail.com

\author{
ACRONYMS \\ RBC: $\quad$ Resin Based Composite \\ SBS: $\quad$ Shear Bond Strength \\ TEGMA: Triethylene Glycol Dimethacrylate
}

\section{INTRODUCTION}

Following clinical placement of the restoration, resin based composites (RBC) undergo significant changes intraorally that result in a gradual deterioration of the material. This degradation almost always results from a combination of chemically-induced or mechanistic processes.

Chemically induced degradation occurs following the action of moisture, salivary enzymes and solvents present in food on the restoration. ${ }^{1}$ Mechanical degradation may be as a result of micro-cracks developing within the restoration, wear of the surface and static fatigue. ${ }^{2}$ The extent and rate of deterioration are dependent on the nature of monomer making up the resin matrix, degree of monomer conversion, structure of the filler particles and the integrity of the silane coupling between the filler and resin matrix. ${ }^{1}$ In vitro, one consequence of aging in artificial saliva is the degeneration of one or possibly all of the components of the RBC: resin matrix, filler particles, silane and initiators. ${ }^{3}$

Thus, old, degraded and defective composite restorations often require some type of repair. Based on available evidence from laboratory and clinical studies, repair of composite restorations may prolong their lifespan while at the same time conserving enamel and dentine. ${ }^{4}$ Repair of the RBC usually involves surface preparation of the old composite, application of an intermediate adhesive followed by addition of the fresh composite onto the prepared surface. $^{5}$

\section{Artificial aging of composites}

In order to simulate in-vitro the clinically degraded restoration, laboratory aging of composite substrate is necessary. A number of methods have been used for this purpose, including thermocycling, immersion in distilled or deionized water, boiling or even in vitro exposure to citric acid and sodium chloride solutions. ${ }^{4}$

Thermocycling continues to be one of the most commonly used methods of aging the RBCs. ${ }^{4,6}$ However, there exists no standardization as to the number of cycles or the duration of time for which the substrate should be thermally cycled. ${ }^{6}$ In addition, concerns have been raised as to the actual clinical relevance of thermocycling when it is used as the sole aging method. ${ }^{6,7}$ 
In order to more accurately simulate the behaviour of composite restorations within the oral environment, a number of different investigators have used artificial saliva to age composite specimens. The composition of artificial saliva used in these studies varies widely. In some instances, commercial preparations of artificial saliva have been used for this purpose ${ }^{8}$ while other investigators manufactured unique solutions incorporating a variety of ingredients specifically for the purpose of aging RBCs.9,10 Moreover, the $\mathrm{pH}$ of these formulations of artificial saliva has been reported to vary between 6 and $6.7 .^{9,11,12}$ Addition of oral bacteria and fungi into the artificial saliva may result in solutions that more closely approximate the role of human saliva in the intraoral degradation of composite restorations. ${ }^{13}$

Hahnel et al. ${ }^{12}$ compared the aging of five brands of composite using three different aging media; 96\% ethanol, distilled water and artificial saliva. In that study, it was determined that aging in distilled water and ethanol was associated with a significant reduction on the Vickers hardness and Flexural strength of the composites. In contrast, aging in artificial saliva was shown to result in a significant increase on the surface roughness of all five composites with little effect on the Vickers hardness. Conversely, Yap et al. $^{14}$ reported dissimilar effects on the mechanical properties when composites were aged in artificial saliva. In three of four brands of composite tested, the surface hardness was found to have increased following storage in a solution of artificial saliva for seven days. At the same time, following examination of sectioned samples with a stereomicroscope, surface degradation was found to occur in all four composites. ${ }^{14}$

A method in which composite blocks were aged in a mixed-species biofilm was recently reported by Rinastiti et al. ${ }^{13}$ This particular biofilm was formulated according to the Zürich biofilm model proposed by Guggenheim et al. ${ }^{15}$ and included a variety of organisms, Streptococcus oralis, Actinomycesnaes/undii, Fusobacterium nucleatum and Candida albicans, together with 5\% foetal bovine serum.

In this case, scanning electron microscope scans showed that composite surfaces exposed to this medium were degraded in a manner similar to that seen on composite blocks left intraorally for eight weeks within a palatal appliance.
It is important to note that the use of artificial saliva simulates only the hydrolytic degradation of composite. ${ }^{3}$ The effect of temperature variation may be tested using thermocycling, while exposure to citric acid reflects attempts to mimic the action of acids within foods. ${ }^{4}$

The cyclic loading of composite from repeated chewing is more difficult to reproduce and is not factored in with these types of aging. The aim of the present study was, therefore, to compare the effect of aging composites in artificial saliva on the shear bond strengths (SBS) between fresh and artificially aged composite. The null hypothesis stated that aging of the composite cylinders in artificial saliva had no effect on the shear bond strength of repaired composite samples.

\section{MATERIALS AND METHODS}

The materials used for this study are listed in Table 1.

Two hundred and forty composite cylinders were made with the aid of a silicone matrix using Filtek Supreme XTE, with each cylinder having a diameter of $5 \mathrm{~mm}$ and height of $5 \mathrm{~mm}$.

To fabricate these samples, composite was applied into the silicone matrix and cured incrementally to depths of $2 \mathrm{~mm}$ until the full height of the sample was achieved.

The curing was carried out using a light-emitting diode (LED) light with a power output $1000 \mathrm{~mW} / \mathrm{cm}^{2}$ (Ellipar Freelight 2, 3M ESPE, St. Paul, MN, USA). The output of this light was verified prior to use with a Cure Rite Light Meter (Dentsply Caulk, Milford, USA) and confirmed after fabrication of every 10 composite cylinders. All the composite cylinders were prepared by a single operator.

One hundred and twenty samples were randomly selected and allocated to the 'non-aging' (group A) while the remainder ( $n=120$ ) were allocated to the 'aging' (group B).

The samples for aging were then immersed in a solution of artificial saliva which had been manufactured according to the method described by van der Bijl \& de Waal ${ }^{16}$ and inoculated with Streptococcus mutans ( $\left.1 \times 10^{6} \mathrm{CFU}\right)$ and Lactobacilli acidophilus ( $\left.1 \times 10^{6} \mathrm{CFU}\right)$. The components of the artificial saliva solution are listed in Table 2.

\begin{tabular}{l|l|l}
$\begin{array}{l}\text { Table 1. List of materials used in the study } \\
\text { MATERIAL }\end{array}$ & DESCRIPTION & MANUFACTURER \\
$\begin{array}{l}\text { Filtek Supreme XTE, shade A3 Body } \\
\text { (A3B) }\end{array}$ & Nano-filled restorative composite & 3M ESPE, St. Paul, MN, USA \\
\hline $\begin{array}{l}\text { Tetric N-Ceram, shade A3 } \\
\text { AdperScotchbond 1XT Adhesive }\end{array}$ & Nano-hybrid restorative composite & Ivoclar Vivadent, Schaan, Liechtenstein \\
\hline Scotchbond Universal Adhesive & Single-bottle universal adhesive & 3M ESPE, St. Paul, MN, USA \\
\hline $\begin{array}{l}\text { Tetric N-Bond Univeral } \\
\text { Ultra-Etch }\end{array}$ & Single-bottle universal adhesive & Ivoclar Vivadent, Schaan, Liechtenstein \\
\hline COJET Sand & Phosphoric acid etchant & Ultradent Products Inc., South Jordan, \\
\hline
\end{tabular}




\begin{tabular}{lr} 
Table 2. Components of the artificial saliva & $\begin{array}{c}\text { Weight in grams or } \\
\text { volume in } \mathrm{mL}\end{array}$ \\
\hline Carboxymethylcellulose & $9.0 \mathrm{~g}$ \\
\hline Potassium chloride & $1.2 \mathrm{~g}$ \\
\hline Sodium chloride & $0.84 \mathrm{~g}$ \\
\hline Magnesium chloride hexahydrate & $0.06 \mathrm{~g}$ \\
\hline Calcium chloride dihydrate & $0.16 \mathrm{~g}$ \\
\hline Dipotassium phosphate & $0.34 \mathrm{~g}$ \\
\hline Sorbitol solution (70\%) & $42.80 \mathrm{~g}$ \\
\hline Methyl p-Hydroxybenzoate & $2.0 \mathrm{~g}$ \\
\hline Solution of egg yellow (1\%) & $2.0 \mathrm{~g}$ \\
\hline Alcohol & $2.5 \mathrm{ml}$ \\
Oil of lemon & $0.4 \mathrm{~g}$ \\
Distilled water & $1000 \mathrm{ml}$
\end{tabular}

The $\mathrm{pH}$ of this solution before inoculation with the bacteria was 6.7. The 120 samples were then stored in an incubator for 28 days at $37^{\circ} \mathrm{C}$. The non-aged composite cylinders were stored at room temperature in a dry and dark storage area for 48 hours before repair.

All the composite cylinders within each of the two groups were embedded in acrylic resin within small sections of PVC pipes. They were then divided into twelve different sub-groups representing six different surface preparation protocols in each group (A1-A6 consisting of the nonaged composite cylinders and B1-B6 consisting of the aged cylinders). Thus, each of the 12 sub-groups had 20 composite cylinders. The six surface preparation protocols are described below.

1. Roughening with a medium-grit diamond bur (MANI Inc., Tochigi, Japan) and application of a single layer Scotchbond 1XT onto the roughened surface.

2. Roughening with a medium-grit diamond bur what type (MANI Inc., Tochigi, Japan), application of 35\% phosphoric acid (Ultra Etch) for 20 seconds, cleaning and drying with a water and air spray. Application of a single layer of Scotchbond 1XT using a microbrush after surface was completely dry.

3. Cleaning of the surface with a water and air spray for 5 seconds. Application of a single layer of Scotchbond Universal onto the dried surface using a microbrush.

4. Surface cleaning with a water and air spray. Application of a single layer of Tetric N-Bond Universal onto the dried surface using a microbrush.

5. No surface treatment. For group A with the non-aged samples, this set represented the positive control while in group B (aged samples) it was used as the negative control.

6. Cleaning of the surface with an air-water spray. Application of COJET Sand for 5 seconds with the tip of the microblaster (Rocatec Junior, 3M ESPE) oriented perpendicular to the composite surface at a distance of $2 \mathrm{~mm}$. The pressure of the microblaster was set at 2 bar in conformity with the instructions from the manufacturer of COJET Sand (3M ESPE).
In all the samples within groups 1, 2, 3, 4 and 6, the respective adhesives were first applied in a single layer using a microbrush, gently air-dried for 5 seconds and then cured for 20 seconds using an LED curing light (Ellipar Freelight 2, 3M ESPE, St. Paul).

After the test samples were subjected to their respective surface treatments, "repair" of all two hundred and forty specimens was carried out using fresh composite. Within each subgroup of 20 samples (A1 to A6 and B1 to B6), 10 samples each were "repaired" by the addition of Filtek Supreme XTE and the remaining 10 samples with Tetric $\mathrm{N}$-Ceram.

All the repair composites were applied and cured in $2 \mathrm{~mm}$ increments using a silicon mould of $3 \mathrm{~mm}$ diameter in the exactly the same manner by which the original composite blocks were prepared. The repair interface was therefore standardized to a bonded surface area of $3 \mathrm{~mm}$ diameter to the bottom composite base, with a height of $4 \mathrm{~mm}$ for the repair composite.

The samples were then mounted on a jig and a shear bond test of the repair interface carried out using a universal testing machine (Zwick Roell International, Germany). For the purpose of the shear bond test, the blade was oriented perpendicular to the long axis of the composite block as close to the repair interface as possible.

A force was then applied onto the blade at a crosshead speed of $0.5 \mathrm{~mm} /$ minute until fracture of the composite occurred. The shear bond test was carried out in accordance with the recommendations on bond strength testing by Heintze. ${ }^{17}$

\section{Data acquisition and analysis}

The force at breaking point was measured in megapascals (MPa) for each sample and recorded on a data collection sheet. The data obtained were then subjected to statistical analysis using computer software, IBM SPSS Statistics version 20 (IBM Corporation, Armonk, U.S.A.).

The effects of each preparation protocol on the shear bond strengths were compared using a one-way Analysis of Variance (ANOVA). Post hoc testing was used to determine where the specific differences between the groups lay. The significance level was set at 0.05.

\section{RESULTS}

The mean SBS values from the aged and nonaged composite samples are shown in Tables 3 and 4 respectively. In five of the six groups of composite repaired with Filtek Supreme XTE, the mean SBS of the non-aged samples was higher than that found in the corresponding aged composites (Figure 1). Only in group 6 (COJET Sand + Scotchbond Universal) was the mean SBS of the aged samples (19.71 MPa) greater than that of the corresponding non-aged composite (18.20 MPa).

Overall, it was found that the combined mean SBS of the aged composites in the six groups repaired with Filtek Supreme XTE (14.36MPa) was $21.1 \%$ lower than that of the non-aged composites. These differences were all found to be statistically significant $(p=0.01)$. 
A similar pattern was observed in the composites repaired with Tetric N-Ceram, where, in four out of the six groups, the aged composites showed a lower SBS than their corresponding non-aged samples (Table 4).

\begin{tabular}{|c|c|c|c|c|}
\hline \multicolumn{2}{|l|}{ Repair protocol } & Mean & $\begin{array}{l}\text { Std. } \\
\text { Deviation }\end{array}$ & $\mathbf{N}$ \\
\hline \multirow{2}{*}{$\begin{array}{l}\text { Bur roughening } \\
+ \text { Scotchbond } 1 \mathrm{XT}\end{array}$} & Non-aged & 18.59 & 4.82 & 10 \\
\hline & Aged & 14.17 & 1.28 & 10 \\
\hline \multirow{2}{*}{$\begin{array}{l}\text { Bur roughening } \\
+\mathrm{PA} \\
+ \text { Scotchbond 1XT }\end{array}$} & Non-aged & 15.85 & 4.67 & 10 \\
\hline & Aged & 14.75 & 1.87 & 10 \\
\hline \multirow{2}{*}{$\begin{array}{l}\text { Scotchbond } \\
\text { Universal }\end{array}$} & Non-aged & 19.05 & 2.70 & 10 \\
\hline & Aged & 15.45 & 1.39 & 10 \\
\hline \multirow{2}{*}{$\begin{array}{l}\text { Tetric N-Bond } \\
\text { Universal }\end{array}$} & Non-aged & 18.16 & 4.58 & 10 \\
\hline & Aged & 15.55 & 3.06 & 10 \\
\hline \multirow{2}{*}{$\begin{array}{l}\text { No surface } \\
\text { preparation or } \\
\text { adhesive }\end{array}$} & Non-aged & 19.45 & 3.57 & 10 \\
\hline & Aged & 6.52 & 2.08 & 10 \\
\hline \multirow{2}{*}{$\begin{array}{l}\text { COJET Sand } \\
\text { + Scotchbond } \\
\text { Universal }\end{array}$} & Non-aged & 18.02 & 3.70 & 10 \\
\hline & Aged & 19.71 & 3.10 & 10 \\
\hline \multirow{2}{*}{$\begin{array}{l}\text { Combined mean } \\
\text { SBS }\end{array}$} & Non-aged & 18.20 & & 60 \\
\hline & Aged & 14.36 & & 60 \\
\hline
\end{tabular}

\begin{tabular}{|c|c|c|c|c|}
\hline Repair protocol & & Mean & $\begin{array}{l}\text { Std. } \\
\text { Deviation }\end{array}$ & $\mathbf{N}$ \\
\hline \multirow{2}{*}{$\begin{array}{l}\text { Bur roughening } \\
+ \text { Scotchbond } 1 \mathrm{XT}\end{array}$} & Non-aged & 16.67 & 3.17 & 10 \\
\hline & Aged & 14.83 & 1.11 & 10 \\
\hline \multirow{2}{*}{$\begin{array}{l}\text { Bur roughening } \\
+ \text { + PA } \\
\text { + Scotchbond 1XT }\end{array}$} & Non-aged & 16.81 & 2.18 & 10 \\
\hline & Aged & 15.09 & 1.93 & 10 \\
\hline \multirow{2}{*}{$\begin{array}{l}\text { Scotchbond } \\
\text { Universal }\end{array}$} & Non-aged & 17.74 & 1.65 & 10 \\
\hline & Aged & 17.83 & 2.06 & 10 \\
\hline \multirow{2}{*}{$\begin{array}{l}\text { Tetric N-Bond } \\
\text { Universal }\end{array}$} & Non-aged & 21.55 & 2.46 & 10 \\
\hline & Aged & 17.35 & 1.49 & 10 \\
\hline \multirow{2}{*}{$\begin{array}{l}\text { No surface } \\
\text { preparation or } \\
\text { adhesive }\end{array}$} & Non-aged & 18.26 & 2.7 & 10 \\
\hline & Aged & 6.89 & 2.32 & 10 \\
\hline \multirow{2}{*}{$\begin{array}{l}\text { COJET Sand } \\
+ \text { Scotchbond } \\
\text { Universal }\end{array}$} & Non-aged & 18.24 & 3.4 & 10 \\
\hline & Aged & 20.83 & 2.87 & 10 \\
\hline \multirow{2}{*}{$\begin{array}{l}\text { Combined mean } \\
\text { SBS }\end{array}$} & Non-aged & 18.21 & & 60 \\
\hline & Aged & 15.47 & & 60 \\
\hline
\end{tabular}

In group 3, the SBS of the aged composites (17.83 MPa) was higher than that of the non-aged group (17.74 MPa) though this difference was not statistically significant.

In group 6 (COJET Sand + Scotchbond Universal), there was a marked difference between the SBS from the aged (20.83 MPa) and the non-aged groups (18.24 MPa). This difference was statistically significant $(p=0.021)$.

As was the case with the samples repaired with Filtek Supreme XTE, the combined mean of the aged composites (15.47 MPa) was $15.04 \%$ lower than that of the non-aged specimens (18.21 MPa).

It was also noted that in both sets of samples repaired with either FiltekSpreme XTE or Tetric N-Ceram, there was a narrow range in the SBS values recorded from the non-aged samples (Figures 1 and 2). This contrasts with the aged samples where a greater variation and range of SBS values was observed.

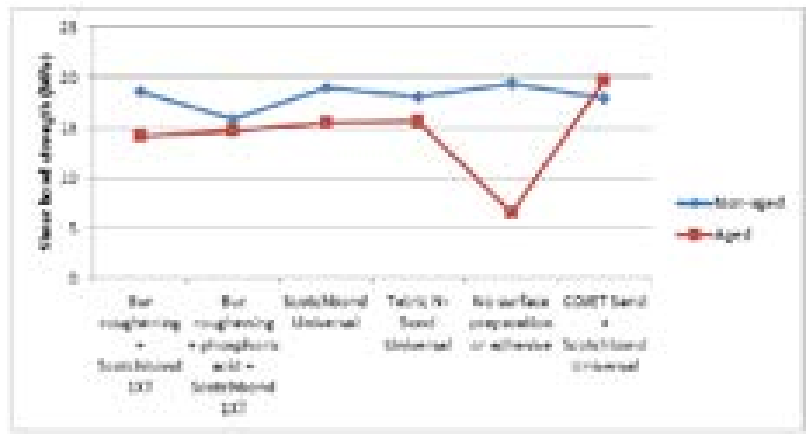

Figure 1. Effect of aging on the mean shear bond strength of composite repaired with Filtek Supreme XTE.

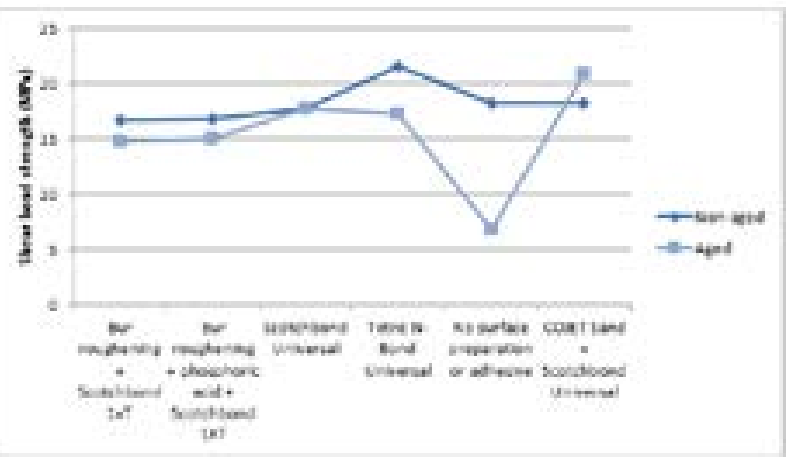

Figure 2. Effect of aging on the mean SBS of composite after repair with Tetric N-Ceram.

\section{DISCUSSION}

In this study, it was found that in general, the bond strength of the repair interface on aged samples was less than that of the corresponding non-aged composites except in the COJET Sand groups, regardless of the repair composite used. When repaired with Filtek Supreme XTE, the mean SBS was $21.1 \%$ lower in this group than in the corresponding non-aged composites.

Similarly, the aged samples repaired with Tetric N-Ceram showed a comparative reduction of $15.04 \%$ in the SBS. The null hypothesis was therefore rejected. The decreased bond strength may be as a result of changes in the surface morphology and chemical composition of the composite following aging in artificial saliva. ${ }^{13}$ 
The negative control was used to isolate the effect of the aging medium and repair protocols on the SBS of the repaired composite. On the other hand, use of the positive control highlighted the repair SBS in relation to the cohesive strength of the RBC.

Irrespective of the repair composite used, four repair methods in the aged samples produced SBS's that were significantly lower than the cohesive strength of the non-aged composites. In contrast, repair with COJET Sand and Scotchbond Universal was associated with a bond strength equivalent to the cohesive strength of the material.

The effectiveness of this repair method may be due to the technique, for sandblasting the COJET Sand particles onto the composite surface results in the silica coating being anchored onto the restoration to a depth of about $15 \mu \mathrm{m}$. When a silane-containing adhesive, such as Scotchbond Universal, is applied onto this composite surface a much stronger micro-mechanical and chemical bond is created that resembles the fillersilane-resin bond within the body of the composite. ${ }^{18}$

Exposure to moisture has been shown to induce hydroIytic degradation in the resin matrix of RBCs. ${ }^{3}$ In a process known as chain scission, the highly crosslinked polymer chains are broken down into shorter fragments. This, coupled with oxidation of the functional groups within the polymer, leads to eventual inactivation of the resin. ${ }^{1}$ As such, the surface of the composite will present a less suitable surface for bonding.

In addition, water sorption may lead to dissociation of the silane-filler bond in the RBC which lowers the mechanical properties of the material and renders its surface more susceptible to degradation. In this process, water uptake into the body of the composite causes the material to become plasticized and lose its fracture strength and elastic modulus. ${ }^{19}$

Hydrophilic monomers such as TEGDMA are responsible for the bulk of water sorption; and as such, RBCs with a high content of these monomers have an increased susceptibility to hydrolytic degradation. ${ }^{20}$

In addition, acids produced by bacteria in the artificial saliva may induce softening and surface swelling of the composite with a subsequent reduction in surface roughness. ${ }^{21}$

\section{Artificial saliva as an aging medium}

Many studies investigating composite repair have used plain water as an aging medium, with or without thermocycling. ${ }^{4}$ Citric acid has also been used in artificial aging of RBCs to simulate the effect of acid in foods and beverages. ${ }^{22}$ However, these two aging methods do not reliably mimic the clinical effect of the oral environment on the composition and surface topography of the RBC.,.$^{6,22}$

Rinastiti et al. ${ }^{13}$ used a biofilm of artificial saliva to age composite samples and compared their surface characteristics with composite specimens worn intraorally in a palatal appliance for 180 days.
They reported that examination with scanning electron micrographs showed a similar pattern of surface degradation for both groups of composites. It therefore seems logical, in a laboratory study which sets out to determine the effect of different repair protocols, to age the composites in artificial saliva first, followed by the routine of the repair protocol as that sequence more accurately simulates the clinical situation.

Thus, the solution of artificial saliva employed in this study was formulated in such a way as to mimic the composition of saliva and its effect on the composite restoration.

Despite the fact that aging composite in artificial saliva appears to reproduce the clinical degradation of composite restorations, there is no standard formula for preparation of this aging medium.

As such, it is difficult to compare results from different studies with confidence. It would therefore be of benefit to have investigators employ solutions of artificial saliva that have been standardized both in composition and manufacturing process.

Additionally, it has been suggested that composites be immersed in artificial saliva immediately after curing. ${ }^{11}$ This reduces the risk of the composite undergoing water sorption from atmospheric air. Furthermore, the temperature, relative humidity, duration of curing ${ }^{11}$ as well as the $\mathrm{pH}$ should be reported to aid in comparison between different studies.

This study evaluated only one aspect of the chemical degradation of composite restorations i.e. the effect of saliva inoculated with bacteria. The effects of temperature variation and acids present in food were not considered in this study. Also, within the oral environment, the restoration is subjected simultaneously to both mechanistic and chemical degradation. ${ }^{23}$

These two factors work in tandem to contribute to the breakdown of the restoration but the effects of this complex interaction were not taken into account when conducting this investigation.

\section{CONCLUSION}

Within the limitations of this study, it may be concluded that composite samples aged in artificial saliva exhibited a significant reduction in bond strength of the repair interface compared with the non-aged samples. The reduction may partly be attributed to a degradation of the bonding surface of the aged composite.

\section{Acknowledgements}

The authors would like to acknowledge 3M ESPE South Africa and Ivoclar Vivadent, Liechtenstein for donating some of the composites and adhesives used in this study.

In addition, we acknowledge Dr. Nicky Basson of the Oral and Dental Research Institute, Faculty of Dentistry, University of the Western Cape for his assistance in formulating the solution of artificial saliva used in this study. 


\section{References}

1. Curtis A. The influence of 'nanocluster' reinforcement on the mechanical properties of a resin-based composite material [Ph.D].University of Birmingham, United Kingdom; 2008. Available from: http://etheses.bham.ac.uk/276/

2. Lohbauer U, Belli R, Ferracane J. Factors involved in mechanical fatigue degradation of dental resin composites. J Dent Res. 2013; 92(7):584-91.

3. Asmussen E, Peutzfeldt A. Two-step curing: influence on conversion and softening of a dental polymer. Dent Mater. 2003; 19(6):466-70.

4. Hickel R, Brüshaver K, llie N. Repair of restorations - criteria for decision making and clinical recommendations. Dent Mater. 2013; 29(1):28-50

5. Irari K. An in-vitro evaluation of repair protocols applied to composite resin [MSc.]. University of the Western Cape; 2016. Available from: https://etd.uwc.ac.za/handle/11394/5043

6. Gale M, Darvell B. Thermal cycling procedures for laboratory testing of dental restorations. J Dent. 1999; 27(2):89-99.

7. Eliades $\mathrm{G}$. Clinical relevance of the formulation and testing of dentine bonding systems. J Dent. 1994; 22(2):73-81.

8. Lee S, Greener E, Menis D. Detection of leached moieties from dental composites in fluid simulating food and saliva. Dent Mater. 1995; 11(5-6):348-53.

9. Wataha J, Rueggeberg F, Lapp C, Lewis J, Lockwood P, Ergle $\mathrm{J}$ et al. In vitro cytotoxicity of resin-containing restorative materials after aging in artificial saliva. Clin Oral Investig. 1999; 3(3):144-9.

10. Mayworm C, Camargo S, Bastian F. Influence of artificial saliva on abrasive wear and microhardness of dental composites filled with nanoparticles. J Dent. 2008; 36(9):703-10.

11. Musanje L, Darvell B. Aspects of water sorption from the air, water and artificial saliva in resin composite restorative materials. Dent Mater. 2003; 19(5):414-22.

12. Hahnel S, Henrich A, Bürgers R, Handel G, Rosentritt M. Investigation of mechanical properties of modern dental composites after artificial aging for one year. Oper Dent. 2010; 35(4):412-9.
13. Rinastiti M, Ozcan M, Siswomihardjo W, Busscher $H$, van der Mei $\mathrm{H}$. Effect of biofilm on the repair bond strengths of composites. J Dent Res. 2010; 89(12):1476-81.

14. Yap A, Tan S, Wee S, Lee C, Lim E, Zeng K. Chemical degradation of composite restoratives. J Oral Rehabil. 2008; 28(11):1015-21.

15. Guggenheim B, Guggenheim M, Gmür R, Giertsen E, Thurnheer T. Application of the Zürich Biofilm Model to problems of Cariology. Caries Res. 2004;38(3):212-22.

16. van der Bijl P, de Waal J. Preparation and clinical evaluation of a high viscosity saliva substitute. J Dent Assoc S Afr. 1994; 49(6):299-303.

17. Heintze S. Clinical relevance of tests on bond strength, microleakage and marginal adaptation. Dent Mater. 2013; 29(1):59-84.

18. Rinastiti M, Özcan M, Siswomihardjo W, Busscher $H$. Immediate repair bond strengths of microhybrid, nanohybrid and nanofilled composites after different surface treatments. J Dent. 2010; 38(1):29-38.

19. Lohbauer U, Frankenberger R, Krämer N, Petschelt A. Time-dependent strength and fatigue resistance of dental direct restorative materials. J Mater Sci Mater Med. 2003; 14(12):1047-53.

20. Musanje L, Shu M, Darvell B. Water sorption and mechanical behaviour of cosmetic direct restorative materials in artificial saliva. Dent Mater. 2001; 17(5):394-401.

21. Asmussen E. Softening of BISGMA-based polymers by ethanol and by organic acids of plaque. Eur Jour Oral Sci. 1984; 92(3):257-61.

22. Özcan M, Barbosa S, Melo R, Galhano G, Bottino M. Effect of surface conditioning methods on the microtensile bond strength of resin composite to composite after aging conditions. Dent Mater. 2007; 23(10):1276-82.

23. Carvalho F, Sampaio C, Fucio S, Carlo H, Correr-Sobrinho L, Puppin-Rontani R. Effect of chemical and mechanical degradation on surface roughness of three glass ionomers and a nanofilled resin composite. Oper Dent. 2012; 37(5):509-17. 